\title{
A zero-infinity law for well-approximable points in Julia sets
}

\author{
RICHARD HILL $\dagger$ and SANJU L. VELANI $\$$ \\ $\dagger$ Department of Mathematics, University College London, Gower Street, \\ London WC1E 6BT, UK \\ (e-mail: the.richard.hill@bigfoot.com) \\ $\ddagger$ Department of Mathematics, Queen Mary, University of London, Mile End Road, \\ London E1 4NS, UK \\ (e-mail: s.velani@qmw.ac.uk)
}

(Received 20 May 2001 and accepted in revised form 20 December 2001)

In memory of Noel Baker

Abstract. Let $T: J \rightarrow J$ be an expanding rational map of the Riemann sphere acting on its Julia set $J$ and $f: J \rightarrow \mathbb{R}$ denote a Hölder continuous function satisfying $f(x)>\log \left|T^{\prime}(x)\right|$ for all $x$ in $J$. Then for any point $z_{0}$ in $J$ define the set $D_{z_{0}}(f)$ of 'well-approximable' points to be the set of points in $J$ which lie in the Euclidean ball

$$
B\left(y, \exp \left(-\sum_{i=0}^{n-1} f\left(T^{i} y\right)\right)\right)
$$

for infinitely many pairs $(y, n)$ satisfying $T^{n}(y)=z_{0}$. In our 1997 paper, we calculated the Hausdorff dimension of $D_{z_{0}}(f)$. In the present paper, we shall show that the Hausdorff measure $\mathcal{H}^{s}$ of this set is either zero or infinite. This is in line with the general philosophy that all 'naturally' occurring sets of well-approximable points should have zero or infinite Hausdorff measure.

\section{Introduction}

In [4], we formulated the following general problem. Consider a metric space $J$ equipped with a Borel probability measure $m$. If $T: J \rightarrow J$ is measure preserving and ergodic, we know by the ergodic theorem that for any ball $B$ of positive $m$-measure, the subset

$$
\left\{z \in J: T^{n}(z) \in B \text { for infinitely many } n \in \mathbb{N}\right\}
$$

of $J$ has full $m$-measure. This means that the trajectories of $m$-almost all points will go through the ball $B$ infinitely often. A natural question to ask is what happens if the ball $B$ $\S$ Royal Society Research Fellow. 
shrinks with time. More precisely, if at time $n$ we have a ball $B\left(z_{0}, \operatorname{rad}(n)\right)$ centred at a point $z_{0} \in J$ of $\operatorname{radius} \operatorname{rad}(n)(\operatorname{rad}(n) \rightarrow 0$ as $n \rightarrow \infty)$, then what kind of properties does the set $W$ of points $z$ have, whose images $T^{n}(z)$ are in $B\left(z_{0}, \operatorname{rad}(n)\right)$ for infinitely many $n$ ? These points can be thought of as trajectories which hit a shrinking target infinitely often and are called 'well approximable' with respect to the function 'rad', in analogy to those in the classical theory of Diophantine approximation.

In [4], we considered a special case of the above general 'shrinking target' problem in which $T$ is an expanding rational map of the Riemann sphere $\overline{\mathbb{C}}=\mathbb{C} \cup\{\infty\}$ and $J=J(T)$ is its Julia set. By the definition of expanding, there exists a constant $\lambda>1$ and an integer $p \geq 1$ such that

$$
\left|\left(T^{p}\right)^{\prime}(z)\right| \geq \lambda \quad \text { for all } z \in J
$$

where $T^{\prime}$ is the derivative of $T$. For such maps, it is known (see [8]) that $J$ is not the whole of $\overline{\mathbb{C}}$ and we may and will assume that $\infty \notin J$. Thus we can think of $J$ as a metric space with the usual metric on $\mathbb{C}$. Specifically, for any $\tau>0$ and $z_{0} \in J$ we considered the sets

$$
W_{z_{0}}^{\bullet}(\tau):=\left\{z \in J: T^{n}(z) \in B\left(z_{0},\left|\left(T^{n}\right)^{\prime}(z)\right|^{-\tau}\right) \text { for infinitely many } n \in \mathbb{N}\right\}
$$

and

$$
W_{z_{0}}(\tau):=\left\{z \text { in } J: T^{n}(z) \in B\left(z_{0}, e^{-n \tau}\right) \text { for infinitely many } n \in \mathbb{N}\right\}
$$

which we referred to as the 'local' and 'global' well-approximable sets, respectively. Here the backward orbit of a selected point $z_{0}$ in $J$ corresponds to the rationals in the classical set $W(\tau):=\left\{x \in \mathbb{R}:|x-p / q| \leq q^{-\tau}\right.$ for infinitely many rationals $\left.p / q\right\}$ of well approximable numbers. For Julia sets associated with rational maps, we proved [4] the following analogue of the Jarník-Besicovitch Theorem in the classical theory of metric Diophantine approximation.

THEOREM 1. For the local set one has $\operatorname{dim} W_{z_{0}}^{\bullet}(\tau)=\delta /(1+\tau)$, where $\delta$ is the Hausdorff dimension of $\mathrm{J}$.

In [4], we also obtained a partial result on the dimension of the global set $W_{z_{0}}(\tau)$. However, the breakthrough in calculating the dimension of this set came by considering the following generalization. Let $f: J \rightarrow \mathbb{R}^{\geq 0}$ denote a Hölder continuous function satisfying

$$
f(z)>\log \left|T^{\prime}(z)\right| \quad \text { for all } z \in J,
$$

and write $f_{n}(x)$ for the $n$th ergodic sum, that is

$$
f_{n}(x):=\sum_{i=0}^{n-1} f\left(T^{i} x\right) .
$$

Then define the set $D_{z_{0}}(f)$ of well-approximable points to be the set of points in $J$ which lie in the ball

$$
B\left(y, \exp \left(-f_{n}(y)\right)\right)
$$

for infinitely many pairs $(y, n)$ with $T^{n}(y)=z_{0}$. In [5] we proved the following. 
THEOREM 2. The set $D_{z_{0}}(f)$ has Hausdorff dimension $\alpha$, where $\alpha$ is the unique positive number satisfying the pressure equation

$$
P(T,-\alpha \cdot f)=0
$$

Here $P(-,-)$ denotes the topological pressure.

It is easy to verify (see $\S 1.3$ in [5]) that the 'local' set corresponds to $D_{z_{0}}(f)$ with $f(z)=(1+\tau) \log \left|T^{\prime}(z)\right|$ whilst the 'global' set corresponds to $D_{z_{0}}(f)$ with $f(z)=$ $\log \left|T^{\prime}(z)\right|+\tau$. This solves the problem of calculating $\operatorname{dim} W_{z_{0}}(\tau)$. The theorem may be viewed as an extension of the Bowen-Manning-McCluskey formula, which states that $P\left(T,-\delta \log \left|T^{\prime}\right|\right)=0$. In [5], we also demonstrated an unexpected link between the dimension results for $D_{z_{0}}(f)$ and the dimension of exceptional sets arising from points with 'badly behaved' ergodic averages. In short, given an ergodic measure $m$ on $J$, these are points $z$ in $J$ at which the ergodic average $\lim _{n \rightarrow \infty} n^{-1} f_{n}(z)$ of $f$ does not tend to the expected limit $\int_{J} f d m$. In turn, Falconer [3] has recently shown a rather elegant connection between the dimension results for $D_{z_{0}}(f)$ and the multifractal spectrum associated with the dynamical system $T: J \rightarrow J$. Also, the dimension result for the local set has recently been extended to parabolic rational maps [7]. This concludes our brief overview of recent developments and various connections. Returning to the main theme, it follows from the definition of $s$-dimensional Hausdorff measure $\mathcal{H}^{s}$ that

$$
\mathcal{H}^{s}\left(D_{z_{0}}(f)\right)= \begin{cases}0 & \text { if } s>\alpha \\ \infty & \text { if } s<\alpha .\end{cases}
$$

However, if $s=\alpha$ then $\mathcal{H}^{s}\left(D_{z_{0}}(f)\right)$ may be zero or infinite, or may satisfy

$$
0<\mathcal{H}^{s}\left(D_{z_{0}}(f)\right)<\infty
$$

In this paper, we shall prove that the latter is impossible.

THEOREM 3. Let $\alpha$ be Hausdorff dimension of $D_{z_{0}}(f)$. Then the $\alpha$-dimensional Hausdorff measure of $D_{z_{0}}(f)$ is either zero or infinity.

Note that if one sets $f=\log \left|T^{\prime}\right|$, the set $D_{z_{0}}(f)$ has full $\delta$-dimensional Hausdorff measure in $J$, where $\delta$ denotes the Hausdorff dimension of $J$. However, for expanding rational maps, $J$ has finite positive $\delta$-dimensional Hausdorff measure and so clearly the theorem does not extend to this case. In fact, the condition $f>\log \left|T^{\prime}\right|$ everywhere on $J$ guarantees that $\mathcal{H}^{\delta}\left(D_{z_{0}}(f)\right)=0$ - see the Appendix.

In the language of geometric measure theory, Theorem 3 simply states that the sets $D_{z_{0}}(f)$ are not $s$-sets. This is a well-known fact for the analogous, classical sets of wellapproximable real numbers. In fact, in the classical set-up the result is a consequence of a much stronger statement whose proof is very technical and rather intricate (see [2] and references within) - there seems to be no direct approach. However, by making use of the geometry of the Julia set and the existence of generalized conformal measures, we are able to give a direct and, in some sense, a 'natural' proof of the statement for expanding rational maps. 
2. Conformal measures

We shall write $\mathcal{H}^{s}$ for the $s$-dimensional Hausdorff measure. Note that the $s$-dimensional Hausdorff measure is $s$-conformal; that is, if $T$ is injective on some set $X \subset J$ then

$$
\mathcal{H}^{s}(T X)=\int_{X}\left|T^{\prime}(x)\right|^{s} d \mathcal{H}^{s}(x) .
$$

Recall that a function $f: J \rightarrow \mathbb{R}$ is said to be Hölder continuous if and only if there is a constant $C$ satisfying the following condition. For any ball $B$ in $J$ and any natural number $n$ such that $T^{n}$ is injective on $B$, one has for all $x, y$ in $B \cap J$

$$
\left|f_{n}(x)-f_{n}(y)\right| \leq C .
$$

The constant $C$ will be referred to as a distortion constant for the function $f$.

We need the following powerful result from complex analysis (see [6]).

KöBe Distortion Theorem. Let $\Delta \subset \overline{\mathbb{C}}$ be a topological disc with boundary containing at least two points and let $V \subset \Delta$ be compact. Then there exists a constant $C(\Delta, V)$ such that for any univalent holomorphic function $U: \Delta \rightarrow \mathbb{C}$ the following inequality is satisfied,

$$
\sup _{x, y \in V} \frac{\left|U^{\prime}(x)\right|}{\left|U^{\prime}(y)\right|} \leq C(\Delta, V)
$$

This implies that the function $\log \left|T^{\prime}\right|$ on $J$ is Hölder continuous.

A more general class of conformal measures was introduced by Denker and Urbański in [1] and numerous other papers. If $f: J \rightarrow \mathbb{R}$ is Hölder continuous, then a measure $v$ on $J$ is said to be $f$-conformal if and only if the following holds. For all measurable subsets $X$ of $J$ on which $T$ is injective:

$$
v(T X)=\int_{X} \exp (f(x)) d v(x) .
$$

In this notation, $\mathcal{H}^{s}$ is $s \log \left|T^{\prime}\right|$-conformal. Denker and Urbański have proved (see [1]) the following theorem.

THEOREM 4. Let T, $f$ and $\alpha$ be as in Theorem 2. Then there is a unique non-atomic $\alpha \cdot f$-conformal probability measure on $J$.

From now on we shall refer to the unique $\alpha \cdot f$-conformal probability measure as $\nu$. We recall the following fact concerning conformal measures.

Lemma 1. Let $T^{n}$ be injective on a ball $B$ in $J$. Then one has

$$
v\left(T^{n} B\right) \asymp \exp \left(\alpha f_{n}(B)\right) v(B)
$$

where $f_{n}(B)$ is the value of $f_{n}$ at any point of $B$ and the implied constants are independent of $B$ and $n$. Similarly one has for any measurable subset $A \subset B$

$$
\mathcal{H}^{\alpha}\left(T^{n} A\right) \asymp\left|\left(T^{n}\right)^{\prime}(B)\right|^{\alpha} \mathcal{H}^{\alpha}(A) .
$$

Proof. This follows from the transformation formula (1) iterated $n$ times, combined with the Hölder continuity of the functions $f$ and $\log \left|T^{\prime}\right|$. 
Next, we state a useful formula for the $v$ measure of an arbitrary ball. For any ball $B$ in $J$, we shall write $n_{0}(B)$ for the largest natural number $n$ for which $T^{n}$ is injective on $B$. Using the fact that $T$ is expanding one may show (see, for example, Lemma 4 of [5]) that there is an $N \in \mathbb{N}$ such that for any ball $B$ in $J$,

$$
J \subset T^{n_{0}(B)+N} B .
$$

This fact together with the previous lemma gives the following lemma.

LEMMA 2. For any ball $B$ one has $v(B) \asymp \exp \left(-\alpha f_{n_{0}(B)}(B)\right)$.

\section{Proof of Theorem 3}

Define the Hölder continuous function $g: J \rightarrow \mathbb{R}^{>0}$ by

$$
g(x):=f(x)-\log \left|T^{\prime}(x)\right| .
$$

For means of calculation we shall introduce the following sets for $C>0$ :

$$
E(C):=\left\{x \in J: T^{n}(x) \in B\left(z_{0}, C \exp \left(-g_{n}(x)\right)\right) \text { for infinitely many } n \in \mathbb{N}\right\}
$$

where $g_{n}$ is the $n$th ergodic sum of $g$. It follows from the Köbe Distortion Theorem that there is a constant $C>1$ such that

$$
E\left(C^{-1}\right) \subset D_{z_{0}}(f) \subset E(C) .
$$

Therefore, in order to prove Theorem 3 it is sufficient to show that either $E(C)$ has zero Hausdorff measure for all $C$, or that $E(C)$ has infinite Hausdorff measure for all $C$.

LEMMA 3. For $x \in J$, one has $x \in E(C)$ if and only if $T(x) \in E\left(C e^{-g(x)}\right)$.

Proof. Let $x \in E(C)$. This means that $T^{n}(x) \in B\left(z_{0}, C \exp \left(-g_{n}(x)\right)\right)$ for infinitely many natural numbers $n$. This is equivalent to $T^{n-1}(T x) \in B\left(z_{0}, C \exp \left(-g_{n-1}(T x)-g(x)\right)\right)$ for infinitely many natural numbers $n$. Replacing $n-1$ by $n$ in this we obtain $T(x) \in$ $E(C \exp (-g(x)))$.

LEMma 4. For $x \in J$ one has $x \in E(C)$ if and only if $T^{n}(x) \in E\left(\exp \left(-g_{n}(x)\right) C\right)$.

Proof. This follows from Lemma 3 by induction on $n$.

LEMMA 5. For any $a>0$ we have $\mathcal{H}^{\alpha}(E(a C)) \asymp \mathcal{H}^{\alpha}(E(C))$, where the implied constants depend on a, but not on $C$.

Proof. Suppose $a<1$. Then clearly $\mathcal{H}^{\alpha}(E(a C)) \leq \mathcal{H}^{\alpha}(E(C))$. On the other hand, by the conformality of Hausdorff measure (see Lemma 1) and the fact that $T$ is expanding, we have that $\mathcal{H}^{\alpha}\left(T^{m}(E(C))\right) \gg \mathcal{H}^{\alpha}(E(C))$ - here the implied constant depends on $m$. As $g(z)>0$ everywhere on the compact set $J$, there is an $\epsilon>0$ such that $g(z)>\epsilon$. Thus $g_{m}(z)>m \epsilon$, which together with Lemma 4 implies that $T^{m}(E(C)) \subset E(C \exp (-m \epsilon))$. Hence

$$
\mathcal{H}^{\alpha}(E(C)) \ll \mathcal{H}^{\alpha}(E(C \exp (-m \epsilon))),
$$

and choosing $m$ such that $\exp (-m \epsilon) \leq a \leq \exp (-(m-1) \epsilon)$ completes the proof when $a<1$. For the case when $a>1$, start by considering $\mathcal{H}^{\alpha}\left(T^{m}(E(a C))\right)$ and repeat the above argument with obvious modifications. 
LEMMA 6. For any ball $B=B(z, r)$ centred on $J$ we have

$$
\mathcal{H}^{\alpha}(B \cap E(C)) \asymp\left|\left(T^{n_{0}(B)}\right)^{\prime}(z)\right|^{-\alpha} \mathcal{H}^{\alpha}\left(E\left(\exp \left(-g_{n_{0}(B)}(z)\right) C\right)\right) .
$$

The implied constants are independent of $B$ and $C$.

Proof. Let $n_{0}=n_{0}(B(z, r))$. By Lemma 1 we have

$$
\mathcal{H}^{\alpha}(B \cap E(C)) \asymp\left|\left(T^{n_{0}}\right)^{\prime}(z)\right|^{-\alpha} \mathcal{H}^{\alpha}\left(T^{n_{0}}(B \cap E(C))\right) .
$$

By Lemma 4 we have

$$
T^{n_{0}}(B \cap E(C)) \subset E\left(\exp \left(-g_{n_{0}}(z)+a\right) C\right),
$$

where $a$ is a distortion constant for $g$. Therefore,

$$
\mathcal{H}^{\alpha}(B \cap E(C)) \ll\left|\left(T^{n_{0}}\right)^{\prime}(z)\right|^{-\alpha} \mathcal{H}^{\alpha}\left(E\left(\exp \left(-g_{n_{0}}(z)+a\right) C\right)\right) .
$$

By Lemma 5 this implies

$$
\mathcal{H}^{\alpha}(B \cap E(C)) \ll\left|\left(T^{n_{0}}\right)^{\prime}(z)\right|^{-\alpha} \mathcal{H}^{\alpha}\left(E\left(\exp \left(-g_{n_{0}}(z)\right) C\right)\right) .
$$

On the other hand, there is a constant $N$ such that $T^{n_{0}+N}(B) \supset J$. This implies

$$
\begin{aligned}
\mathcal{H}^{\alpha}(B \cap E(C)) & \gg\left|\left(T^{n_{0}+N}\right)^{\prime}(z)\right|^{-\alpha} \mathcal{H}^{\alpha}\left(T^{n_{0}+N}(B \cap E(C))\right) \\
& \gg\left|\left(T^{n_{0}}\right)^{\prime}(z)\right|^{-\alpha} \mathcal{H}^{\alpha}\left(E\left(\exp \left(-g_{n_{0}+N}(z)-a\right) C\right)\right),
\end{aligned}
$$

and again by Lemma 5 we have

$$
\mathcal{H}^{\alpha}(B \cap E(C)) \gg\left|\left(T^{n_{0}}\right)^{\prime}(z)\right|^{-\alpha} \mathcal{H}^{\alpha}\left(E\left(\exp \left(-g_{n_{0}}(z)\right) C\right)\right) .
$$

To prove the next lemma, we will use a carefully chosen cover of the Julia set $J$ which we now describe. Let $I$ be the set of pairs $(y, n) \in J \times \mathbb{N}$ such that $T^{n}(y)=z_{0}$. Suppose we have $c_{1}, C>0$ and set $I\left(C, c_{1}\right)=\left\{(y, n) \in I:\left|C-\log g_{n}(y)\right|<c_{1}\right\}$. It is known that if $c_{1}$ is sufficiently large (depending only on $J$ and $f$ ) then for any $C>0$ the following is a cover of $J$ :

$$
\mathcal{C}(C)=\left\{B\left(y, c_{1}\left|\left(T^{n}\right)^{\prime}(y)\right|^{-1}\right):(y, n) \in I\left(C, c_{1}\right)\right\} .
$$

We shall fix $c_{1}$ sufficiently large so that $\mathcal{C}(C)$ is a cover. There is a constant $N$ depending only on $J, f$ and $c_{1}$ such that no point of $J$ is in more than $N$ of the balls in the cover $\mathcal{C}(C)$. These statements are contained in Lemma 8 of [5]. The upshot of this is that for any measure $\mu$ on $J$ and any measurable subset $A \subseteq J$, we have

$$
\mu(A) \asymp \sum_{(y, n) \in I\left(C, c_{1}\right)} \mu\left(A \cap B\left(y, c_{1}\left|\left(T^{n}\right)^{\prime}(y)\right|^{-1}\right)\right) .
$$

The implied constants are independent of $\mu$ and $C$ (in fact, the implied constants are 1 and $N$ ).

Lemma 7. Let $B$ be a ball in $J$. Then $\mathcal{H}^{\alpha}(B \cap E(C)) \asymp C^{\alpha} \mathcal{H}^{\alpha}(B \cap E(1))$. 
Proof. By Lemma 6 it is sufficient to prove the lemma in the case $B=J$. By (2) we have

$$
\mathcal{H}^{\alpha}(E(C)) \asymp \sum_{(y, n) \in I\left(C, c_{1}\right)} \mathcal{H}^{\alpha}\left(B\left(y, c_{1}\left|\left(T^{n}\right)^{\prime}(y)\right|^{-1}\right) \cap E(C)\right) .
$$

For the moment we shall concentrate on one of the balls $B=B\left(y, c_{1}\left|\left(T^{n}\right)^{\prime}(y)\right|^{-1}\right)$ in the cover $\mathcal{C}(C)$. Note that $n_{0}(B)=n+O(1)$ (see Lemma 5 of [5]). Therefore, by Lemma 6 we have

$$
\mathcal{H}^{\alpha}(B \cap E(C)) \asymp\left|\left(T^{n}\right)^{\prime}(y)\right|^{-\alpha} \mathcal{H}^{\alpha}\left(E\left(C e^{-g_{n}(y)}\right)\right) .
$$

Now by Lemma 5 as $\exp \left(g_{n}(y)\right) \asymp C$, we have

$$
\mathcal{H}^{\alpha}(E(C) \cap B) \asymp\left|\left(T^{n}\right)^{\prime}(y)\right|^{-\alpha} \mathcal{H}^{\alpha}(E(1)) .
$$

Again since $\exp \left(g_{n}(y)\right) \asymp C$, by Lemma 2 we have

$$
\left|\left(T^{n}\right)^{\prime}(y)\right|^{-\alpha} \asymp C^{\alpha} \exp \left(-\alpha f_{n}(y)\right) \asymp C^{\alpha} \nu(B) .
$$

Summing this over the balls $B$ in our cover $\mathcal{C}(C)$ we obtain

$$
\mathcal{H}^{\alpha}(E(C)) \asymp C^{\alpha} \mathcal{H}^{\alpha}(E(1)) \sum_{(y, n) \in I\left(C, c_{1}\right)} v\left(B\left(y, c_{1}\left|\left(T^{n}\right)^{\prime}(y)\right|^{-1}\right)\right) .
$$

Now, using (2) in the opposite direction we have

$$
\mathcal{H}^{\alpha}(E(C)) \asymp C^{\alpha} \mathcal{H}^{\alpha}(E(1)) v(J)=C^{\alpha} \mathcal{H}^{\alpha}(E(1)) .
$$

This proves the lemma.

For a ball $B$ in $J$ we shall use the notation

$$
m(B):=\mathcal{H}^{\alpha}(B \cap E(1)) .
$$

LEMMA 8. For any ball $B$ in $J$ we have $m(B) \asymp \mathcal{H}^{\alpha}(E(1)) v(B)$.

Proof. Given $B=B(z, r)$, let $n_{0}=n_{0}(B(z, r))$. By Lemma 6 with $C=1$ and Lemma 7 with $B=J$, we have

$$
\begin{aligned}
m(B) & \asymp\left|\left(T^{n_{0}}\right)^{\prime}(z)\right|^{-\alpha} \mathcal{H}^{\alpha}\left(E\left(\exp \left(-g_{n_{0}}(z)\right)\right)\right) \\
& \asymp \exp \left(-\alpha f_{n_{0}}(z)\right) \mathcal{H}^{\alpha}(E(1)) .
\end{aligned}
$$

The lemma now follows on applying Lemma 2.

During the proof of the next result we will require the following fact (see Corollary 1 of [5]):

$$
\sum_{y: T^{n}(y)=z_{0}} \exp \left(-\alpha f_{n}(y)\right) \asymp 1 .
$$

PROPOSITION 1. $v(E(1))=0$.

Proof. For $C$ sufficiently large the set $E(1)$ is contained in the limsup of the sets $A(n)$ defined by

$$
A(n):=\bigcup_{y: T^{n}(y)=z_{0}} B\left(y, C \exp \left(-f_{n}(y)\right)\right) .
$$


Therefore, in view of the Borel-Cantelli Lemma it is sufficient to show that $\sum_{n} v(A(n))$ converges. By Lemma 1 we have

$$
v(A(n)) \ll \sum_{y: T^{n}(y)=z_{0}} \exp \left(-\alpha f_{n}(y)\right) v\left(B\left(z_{0}, C^{2} \exp \left(-g_{n}(y)\right)\right)\right) .
$$

As $g(z)>0$ everywhere on the compact set $J$, there is an $\epsilon>0$ such that $g(z)>\epsilon$. Thus $g_{n}(z)>n \epsilon$. This together with the above fact implies that

$$
v(A(n)) \ll v\left(B\left(z_{0}, C^{2} \exp (-n \epsilon)\right)\right) \sum_{y: T^{n}(y)=z_{0}} \exp \left(-\alpha f_{n}(y)\right) \ll v\left(B\left(z_{0}, C^{2} \exp (-n \epsilon)\right)\right) .
$$

Next, note that there is a $\rho>0$ such that $T$ is injective on any ball $B$ centred on $J$ of radius $r<\rho$. Therefore,

$$
n_{0}(B(z, r)) \geq \frac{\log (\rho / r)}{\log \left\|T^{\prime}\right\|} \gg-\log (r),
$$

where $\left\|T^{\prime}\right\|$ is the supremum norm of $T^{\prime}$ on the $\delta$-neighbourhood of $J$. Therefore, by Lemma 2 and the fact that $T$ is expanding, we have

$$
\log v(B(z, r)) \ll-n_{0}(B(z, r)) \ll \log (r) .
$$

We now have

$$
\log v(A(n)) \ll \log \left(C^{2} \exp (-n \epsilon)\right) \ll-n .
$$

By the ratio test it follows that $\sum v(A(n))$ converges.

Proof of Theorem 3. Choose $\epsilon>0$. In view of the above proposition, there is a cover $\left\{B_{i}\right\}$ of $E(1)$ such that

$$
\sum_{i} v\left(B_{i}\right)<\epsilon
$$

This implies by Lemma 8 that

$$
\sum_{i} m\left(B_{i}\right) \ll \epsilon \mathcal{H}^{\alpha}(E(1))
$$

Now $\left\{B_{i}\right\}$ is a cover of $E(1)$, so we also have that

$$
\mathcal{H}^{\alpha}(E(1)) \leq \sum_{i} m\left(B_{i}\right)
$$

Therefore,

$$
\mathcal{H}^{\alpha}(E(1)) \ll \epsilon \mathcal{H}^{\alpha}(E(1)) .
$$

Since $\epsilon>0$ is arbitrary, this implies that $\mathcal{H}^{\alpha}(E(1))$ is either zero or infinite. By Lemma 5 we have $\mathcal{H}^{\alpha}(E(C))=\mathcal{H}^{\alpha}(E(1))$ for all $C>0$ which completes the proof of the theorem.

\section{A. Appendix}

We end by establishing the remarks made following the statement of Theorem 3 in the introduction. As always, $\delta$ is the Hausdorff dimension of $J$. 
Lemma A.1.

(i) If $f=\log \left|T^{\prime}\right|$, then

$$
0<\mathcal{H}^{\delta}\left(D_{z_{0}}(f)\right)<\infty .
$$

(ii) If $f>\log \left|T^{\prime}\right|$ everywhere on $J$, then

$$
\mathcal{H}^{\delta}\left(D_{z_{0}}(f)\right)=0 .
$$

The proof of the lemma makes use of certain well-known facts which we now summarize. For an expanding rational map $T$, the $\delta$-conformal measure $v$ supported on $J$ is equivalent to $\delta$-dimensional Hausdorff measure $\mathcal{H}^{\delta}$ and so $\mathcal{H}^{\delta}(J)$ is positive and finite. Furthermore, $v$ has a unique (and hence ergodic) equivalent $T$-invariant probability measure, $\mu$ which is the unique equilibrium state for $T$ and $-\delta \log \left|T^{\prime}\right|$. Thus, for any measurable subset $X$ of $J$ we have that

$$
\mu(X) \asymp \mathcal{H}^{\delta}(X) .
$$

Also

$$
\mu(B(x, r)) \asymp r^{\delta}
$$

for any ball $B$ with centre $x$ in $J$ and radius $r<r_{0}$. For further details see $[4,8]$.

Proof. We work with the $T$-invariant probability measure $\mu$. Recall, that there is a constant $C>1$ such that

$$
E\left(C^{-1}\right) \subset D_{z_{0}}(f) \subset E(C) .
$$

If $f=\log \left|T^{\prime}\right|$, then $E\left(C^{-1}\right)$ consists of points $x$ in $J$ whose forward orbit $T^{n}(x)$ lands in the fixed ball $B\left(z_{0}, C^{-1}\right)$ infinitely often. Either by Poincaré recurrence or the ergodic theorem, $\mu\left(E\left(C^{-1}\right)\right)=1$. Thus $\mu\left(D_{z_{0}}(f)\right)=1$, which together with (A.1) proves the first part of the lemma.

If $f>\log \left|T^{\prime}\right|$ everywhere on $J$, then as already mentioned above $g_{n}(x)>n \epsilon$ everywhere on $J$. Thus, $E(C) \subset E^{*}(C):=\lim _{\sup _{n \rightarrow \infty}} E_{n}^{*}(C)$ where

$$
E_{n}^{*}(C):=\left\{x \in J: T^{n}(x) \in B\left(z_{0}, C \exp (-n \epsilon)\right)\right\} .
$$

By (A.2) and the fact that $\mu$ is $T$-invariant, we have

$$
\mu\left(E_{n}^{*}(C)\right)=\mu\left(B\left(z_{0}, C \exp (-n \epsilon)\right)\right) \asymp(C \exp (-n \epsilon))^{\delta} .
$$

Hence

$$
\sum_{n=1}^{\infty} \mu\left(E_{n}^{*}(C)\right) \ll \sum_{n=1}^{\infty} \exp (-n \epsilon \delta)<\infty
$$

and so, by the Borel-Cantelli Lemma $\mu\left(E^{*}(C)\right)=0$. Thus $\mu(E(C))=0$, which together with (A.1) completes the proof of the lemma.

\section{REFERENCES}

[1] M. Denker and M. Urbański. Ergodic theory of equilibrium states of rational maps. Nonlinearity 4 (1991), $103-134$. 
[2] H. Dickinson and S. L. Velani. Hausdorff measure and linear forms. J. Reine Angew. Math. 490 (1997), 1-36.

[3] K. J. Falconer. Representation of families of sets by measures, dimension spectra and diophantine approximation. Math. Proc. Camb. Phil. Soc. 128 (2000), 111-121.

[4] R. Hill and S. L. Velani. The ergodic theory of shrinking targets. Invent. Math. 119 (1995), 175-198.

[5] R. Hill and S. L. Velani. Metric Diophantine approximation in Julia sets of expanding rational maps. Inst. Hautes Etudes Sci. Publ. Math. 85 (1997), 193-216.

[6] E. Hille. Analytic Function Theory. Ginn and Company, Boston, MA, 1962

[7] B. O. Stratmann and M. Urbański. Jarník and Julia; a Diophantine analysis for parabolic rational maps. Mathematica Scandinavica, to appear.

[8] D. Sullivan. Conformal dynamical systems. Proc. Conf. on Geometric Dynamics (Rio de Janeiro, 1981) (Lecture Notes in Mathematics, 1007). Springer, pp. 725-752. 